\title{
Single-tube library preparation for degraded DNA
}

Carøe, Christian; Gopalakrishnan, Shyam; Vinner, Lasse ; Mak, Sarah S. T.; Sinding, Mikkel-Holger S.; Samaniego, Jose A.; Wales, Nathan; Sicheritz-Pontén, Thomas; Gilbert, M. Thomas P.

Published in:

Methods in Ecology and Evolution

Link to article, DOI:

$10.1111 / 2041-210 X .12871$

Publication date:

2018

Document Version

Peer reviewed version

Link back to DTU Orbit

Citation (APA):

Carøe, C., Gopalakrishnan, S., Vinner, L., Mak, S. S. T., Sinding, M-H. S., Samaniego, J. A., Wales, N., Sicheritz-Pontén, T., \& Gilbert, M. T. P. (2018). Single-tube library preparation for degraded DNA. Methods in Ecology and Evolution, 9(2). https://doi.org/10.1111/2041-210X.12871

\section{General rights}

Copyright and moral rights for the publications made accessible in the public portal are retained by the authors and/or other copyright owners and it is a condition of accessing publications that users recognise and abide by the legal requirements associated with these rights.

- Users may download and print one copy of any publication from the public portal for the purpose of private study or research.

- You may not further distribute the material or use it for any profit-making activity or commercial gain

- You may freely distribute the URL identifying the publication in the public portal 
MR. CHRISTIAN CARØE (Orcid ID : 0000-0001-9601-6768)

Article type : Research Article

Handling editor: Dr. Susan Johnston

Title: Single-tube library preparation for degraded DNA

Running title: Library preparation for degraded DNA

Authors: Christian Carøe ${ }^{1,2}$, Shyam Gopalakrishnan ${ }^{1}$, Lasse Vinner ${ }^{1}$, Sarah S. T. Mak $^{1}$, Mikkel-Holger S. Sinding ${ }^{1,3}$, José A. Samaniego ${ }^{1}$, Nathan Wales ${ }^{1}$, Thomas Sicheritz-Pontén² , M. Thomas P. Gilbert ${ }^{1,4,5}$

1) University of Copenhagen, Natural History Museum of Denmark, Øster Voldgade 5-7, 1350 Copenhagen, Denmark.

2) Center for Biological Sequence Analysis, Dept. of Bio and Health Informatics, Technical University of Denmark, Building 208, DK-2800 Lyngby, Denmark.

3) Natural History Museum, University of Oslo, PO Box 1172 Blindern, N-0318 Oslo, Norway.

4) Trace and Environmental DNA Laboratory, Department of Environment and Agriculture, Curtin University, Perth, Australia.

5) NTNU University Museum, 7491 Trondheim, Norway.

Correspondence: Christian Carøe, Center for Biological Sequence Analysis, Dept. of Systems Biology, Technical University of Denmark, Building 208, DK-2800

Lyngby; Email: Caroe@cbs.dtu.dk; christiancaroe@snm.ku.dk;

Christiancaroe@gmail.com

This article has been accepted for publication and undergone full peer review but has not been through the copyediting, typesetting, pagination and proofreading process, which may lead to differences between this version and the Version of Record. Please cite this article as doi: $10.1111 / 2041-210 X .12871$

This article is protected by copyright. All rights reserved. 


\section{Abstract}

1) In recent years, massive parallel sequencing has revolutionized the study of degraded DNA, thus enabling the field of ancient DNA to evolve into that of paleogenomics. Despite these advances, the recovery and sequencing of degraded DNA remains challenging due to limitations in the manipulation of chemically damaged and highly fragmented DNA molecules. In particular, the enzymatic reactions and DNA purification steps during library preparation can result in DNA template loss and sequencing biases, affecting downstream analyses. The development of library preparation methods that circumvent these obstacles and enable higher throughput are therefore of interest to researchers working with degraded DNA.

2) In this study, we compare four Illumina library preparation protocols, including two "single-tube" methods developed for this study with the explicit aim of improving data quality and reducing preparation time and expenses. The methods are tested on grey wolf (Canis lupus) museum specimens.

3) We found single-tube protocols increase library complexity, yield more reads that map uniquely to the reference genome, reduce processing time, and may decrease laboratory costs by $90 \%$.

4) Given the advantages of single-tube library preparations, we anticipate these methods will be of considerable interest to the growing field of paleogenomics and other applications investigating degraded DNA.

Key words: Degraded DNA, Illumina sequencing, library preparation, museomics, paleogenomics 


\section{Introduction}

With the advent of massive parallel sequencing, the field of ancient DNA (aDNA) has experienced a revolution in data production, maturing into a discipline commonly termed paleogenomics (Poinar et al. 2006; Shapiro \& Hofreiter, 2014; Heintzman et al. 2015). This revolution has been undertaken with multiple sequencing platforms, including the Roche/454 series, Ion Torrent series, Helicos HeliScope, and ABI Solid series, but the undisputed workhorses of aDNA research are the Illumina instruments (e.g. GAIIx, HiSeq, NextSeq and MiSeq series) (Orlando et al. 2015). All the aforementioned platforms require enzymatic preparation of DNA, including, in most cases, ligation of DNA-based platformspecific adapters. However, these preparations often lead to biases in GCcontent, fragment length, or complexity (Aird et al. 2011; Dabney \& Meyer, 2012; Seguin-Orlando et al. 2013; Head et al. 2014; Van Dijk et al. 2014). These challenges are further magnified in degraded samples, due to low amounts of input DNA, short lengths of the DNA fragments, hydrolytic deamination of cytosine and other chemical damage (reviewed in Dabney et al. 2013b). To overcome these complications in degraded samples, researchers have developed library building and PCR protocols optimized to recover small amounts of DNA and tackle chemical damage (e.g. d'Abbadie et al. 2007; Shapiro, 2008; Heyn et al. 2010; Dabney et al. 2013b; Gansauge and Meyer, 2013). Methodological developments to increase library complexity have principally focused on improving the efficiency of DNA extraction and reducing biases in PCR (Rohland \& Hofreiter, 2007; Dabney et al. 2013a; Allentoft et al. 2015; Damgaard et al. 2015; Gamba et al. 2015). A number of studies have aimed to improve library 
preparation by minimizing template loss or maximizing endogenous DNA inclusion into the library (Maricic \& Pääbo, 2009; Ginolhac et al. 2012; Gansauge \& Meyer, 2013; Bennett et al. 2014; Gansauge \& Meyer, 2014; Fortes \& Paijmans, 2015; Wales et al. 2015).

Currently, three principal methods are used when constructing sequencing libraries from aDNA (reviewed in Orlando et al. 2015).

In the original method for the Illumina platform, Y-shaped adapters are ligated to double-stranded target DNA ensuring all single-stranded library molecules have a P5-P7 (5'-3') adapter configuration (Bentley et al. 2008). This protocol, however, is not well suited for short fragments (here loosely defined as fragments with a length of $\leq 70 \mathrm{bp}$ ) and low amounts of DNA due to a high production of adapter-dimer "background noise" (Bennett et al. 2014). In the second and most common method used for aDNA, blunt-ended adapters are ligated to double-stranded DNA, following a protocol originally developed for the 454 platform (Margulies et al. 2005) and later adapted for the Illumina platform (Meyer \& Kircher, 2010) (Figure 1). This method produces less adapter-dimers, but is suboptimal for degraded DNA due to the necessary use of inter-reaction purifications using e.g. silica spin-columns (see e.g. Briggs \& Heyn, 2012) that hamper throughput and have been shown to result in considerable loss of DNA (Kemp et al. 2014). The third method is unique in its use of single-stranded DNA as starting material (Gansauge \& Meyer, 2013). It has been considered revolutionary for aDNA research because it theoretically allows for the recovery of all DNA molecules in a sample. In this way, the method yields high library complexity and more accurately characterizes chemical damage at both ends of 
each DNA strand (Meyer et al. 2013). In its original form, however, the technique is relatively expensive due to the required quantity and cost of key reagents. In addition, the protocol is relatively time consuming and requires some technical expertise. However, recent modifications of the method have addressed some of these issues and improved the method (Gansauge et al. 2017). In parallel with attempts to optimize library preparations for degraded DNA, researchers have developed novel methods to prepare Illumina libraries with minimal effort and cost from high-quality modern DNA. One of the most promising advances is the introduction of "single-tube" library preparations, which are protocols that eliminate inter-reaction purifications (Neiman et al. 2012; Zheng et al. 2011). Such single-tube designs are made possible by replacement of column-based purifications with heat-inactivation of enzymes. This makes library preparation considerably quicker, with fewer manual manipulations, and reduces economic costs. Such approaches also have the advantage of sidestepping potential DNA loss during purification steps. While immensely useful for modern DNA, single-tube methods could be less suitable for highly degraded DNA because they require incubations typically at $72{ }^{\circ} \mathrm{C}$ (Neiman et al. 2012; Zheng et al. 2011). Although such a step has been implemented in an aDNA protocol (Fortes \& Paijmans, 2015), many ultra-short double-stranded DNA fragments (e.g. at $25 \mathrm{bp}$ ) may denature at $72^{\circ} \mathrm{C}$ depending on the sequence composition (Owczarzy et al. 1997). Thus, in order for degraded DNA to be incorporated into an Illumina-compatible library in a single-tube reaction, incubations would have to be kept at a relatively low temperature. Fortuitously, New England Biolabs (NEB) recently released commercial single- 
tube kits with heat inactivation steps of $65^{\circ} \mathrm{C}$, demonstrate the possibility of inactivation steps lower than $72^{\circ} \mathrm{C}$. Importantly, these kits utilize adapters with a hairpin structure and a uracil base that is excised before library amplification (e.g. NEBNext Ultra E7370 [NEB]). As degraded DNA characteristically contains uracil residues (Briggs et al. 2007), the uracil excision step would detrimentally affect NEBNext Ultra libraries prepared using degraded DNA. Although it is possible to remove uracil bases prior to library preparation (Briggs et al. 2010), paleogeneticists often choose to leave uracil damage patterns intact in order to authenticate the ancient origin of reads (e.g. Ginolhac et al. 2011; Meyer et al. 2016; Skoglund et al. 2014).

For this study, we investigated whether single-tube library preparation protocols could accommodate degraded DNA by using heat inactivation temperatures at $65^{\circ} \mathrm{C}$ and employing the advantages of the displacement adapter approach for the Illumina platform described by Meyer \& Kircher (2010) (Figure 1). We compared four library preparation protocols: i) the widely-used protocol based on blunt-end adapter ligation and spin column purifications between reactions (see e.g. Briggs \& Heyn, 2012; Wales et al. 2015), ii) our novel blunt-end adapter ligation single-tube method, iii) our novel A/T adapter ligation single-tube method, and iv) the commercial aforementioned NEBNext Ultra, which we customized to fit the same displacement-type adapter as used in the other methods. In this way, we tested A/T-ligation against blunt-end ligation and the single-tube approach against the common approach including inter-reaction purifications. 


\section{Methods and materials}

All DNA manipulation until library amplification (PCR) was performed in a dedicated aDNA laboratory at the Centre for GeoGenetics, Natural History Museum of Denmark, University of Copenhagen, Denmark, to limit the risk of contamination with non-degraded or amplified DNA sources.

\section{Sample information}

Eight historic skin samples (90 to 146 years old) of grey wolf (Canis lupus) were included in the study (Supplementary, Table S2). For details on DNA extraction see supplementary material. Average fragment sizes ranged from $\sim 40$ to 184 basepairs, lengths commonly observed in ancient or otherwise degraded samples (Supplementary, Table S2 and Figure S1). For a given sample, all four library preparations started with the same mass of input DNA (ng), with dilutions used to reach the required input volume for a given method. Libraries were produced the same day to minimize bias.

\section{Adapter design}

For the standard blunt-end method (BEMC), we used the adapters described by Meyer \& Kircher (2010). For the single-tube methods however, we designed two additional pairs of adapters - one for blunt-end ligation (BEDC3) and one for A/T-ligation (ATDC3). Although these have similarity to the adapter described by Meyer \& Kircher (2010), they both include a C3 spacer arm at the 3' terminus of the IS3 oligo to counteract unwanted extension and reduce noise from adapterdimer formation in the fill-in step. Furthermore, in a series of experiments we 
tested the possibility of minimizing adapter-dimers by constructing other adapters. These experiments did not produce positive results and are described in the supplementary material. An overview of oligos and adapters is shown in supplementary Table S1. Hybridization of adapter oligos was done following Meyer \& Kircher (2010).

\section{Methods for preparation of sequencing libraries}

A short overview of the library preparation methods can be seen in Table 1 and all methods are based on the setup shown in Figure 1.

Method 1: "ATST", denoting A/T-Ligation-Single-Tube. Libraries were prepared in three sequential reactions: end-repair, adapter ligation, and nick fill-in. Endrepair was performed by mixing the following components in a $0.5 \mathrm{~mL}$ Eppendorf LoBind tube (Eppendorf, Germany): $14 \mu \mathrm{L}$ sample DNA was mixed with $2 \mu \mathrm{L}$ of a mastermix made by combining $0.01 \mu \mathrm{L}$ Taq Polymerase (NEB, cat\#M0273S, 5 U/ $\mu \mathrm{L}$ ), $0.01 \mu \mathrm{L}$ T4 DNA Polymerase (NEB, cat\#M0203S, $3 \mathrm{U} / \mu \mathrm{L}$ ), $0.1 \mu \mathrm{L}$ T4 Polynucleotide Kinase (NEB, cat\#M0201S, $10 \mathrm{U} / \mu \mathrm{L}$ ), $1.6 \mu \mathrm{L}$ 10× T4 DNA Ligase Reaction Buffer (NEB) and $0.3 \mu \mathrm{L}$ dNTP $(25 \mathrm{mM})$ per sample to reach a final reaction volume of $16 \mu \mathrm{L}$. The end-repair reaction was incubated for 30 minutes at $20^{\circ} \mathrm{C}$ followed by 30 minutes at $65^{\circ} \mathrm{C}$. For adapter-ligation, $1 \mu \mathrm{L}$ of ATDC3 adapter $(10 \mu \mathrm{M})$ (Supplementary, Table S1) was added to the same reaction tube and mixed by pipetting, followed by $3 \mu \mathrm{L}$ of mastermix consisting of $2.5 \mu \mathrm{L}$ PEG 4000 (Sigma Aldrich, 50\%), 0.4 $\mu \mathrm{L}$ T4 DNA Ligase Reaction Buffer (10×), and 0.1 $\mu \mathrm{L}$ T4 DNA ligase (NEB, cat\#M0202S, $400 \mathrm{U} / \mu \mathrm{L}$ ). The ligation reaction was 
incubated for 30 minutes at $20^{\circ} \mathrm{C}$. The fill-in step was performed by adding $10 \mu \mathrm{L}$ of mastermix consisting of $0.3 \mu \mathrm{L}$ dNTP $(25 \mathrm{mM}), 3 \mu \mathrm{L}$ Isothermal Amplification Buffer (10×) (NEB), $6.2 \mu \mathrm{L}$ molecular biology grade water, and $0.5 \mu \mathrm{L}$ Bst 2.0 Warmstart Polymerase (NEB, cat\#M0538S, 8U/ $\mu \mathrm{L}$ ). The fill-in reaction was incubated at $65^{\circ} \mathrm{C}$ for 20 minutes in a prewarmed thermocycler, followed by 20 minutes at $80^{\circ} \mathrm{C}$. Following library preparation, the reaction was purified with a MinElute column following the manufacturer's instructions and eluted in $25 \mu \mathrm{L}$ EB.

Method 2: "BEST", denoting Blunt-End-Single-Tube. This protocol was identical to the ATST protocol, except for the exclusion of the Taq Polymerase, and the use of blunt-end adapter BEDC3 (Supplementary, Table S1).

Method 3: "BEMC", denoting Blunt-End-Multi-Column. Libraries were prepared using end-repair, adapter ligation, and nick fill-in reactions supplied by NEBNext kit E6070 (NEB). The following components were mixed in a $0.5 \mathrm{~mL}$ Eppendorf LoBind tube: $21.25 \mu \mathrm{L}$ sample DNA, $2.5 \mu \mathrm{L}$ NEBNext 10× End Repair Reaction Buffer, and 1.25 $\mu \mathrm{L}$ NEBNext End Repair Enzyme Mix. The reaction was incubated for $30 \mathrm{~min}$ at $20^{\circ} \mathrm{C}$, and then purified in a MinElute spin column. Purification was done using $700 \mu \mathrm{L}$ modified PB binding buffer (Allentoft et al. 2015) and centrifugation at $6,000 \times g$. The column was washed with $750 \mu \mathrm{L} \mathrm{PE}$ buffer and spun at $10,000 \times g$, followed by an additional spin for 3 minutes at $17,000 \times g$. DNA was eluted in $16.5 \mu \mathrm{L}$ EB buffer, with incubation at $37^{\circ} \mathrm{C}$ for 10 minutes before collecting DNA at 17,000 $\times g$. For the ligation reaction, $1 \mu \mathrm{L}$ 
Illumina adapters $(10 \mu \mathrm{M})($ Meyer \& Kircher, 2010) were thoroughly mixed with the end-repaired DNA, followed by $5 \mu \mathrm{L} 5 \times$ NEBNext Quick Ligation Reaction Buffer and 2.5 $\mu \mathrm{L}$ Quick T4 DNA Ligase. The reaction was incubated for 30 minutes at $20^{\circ} \mathrm{C}$. Adapter-ligated DNA was purified using a Qiagen QiaQuick spin column as before, except for an elution volume of $21 \mu \mathrm{L}$. The fill-in reaction was performed using 2.5 $\mu \mathrm{L}$ 10× NEBNext Adapter Fill-in Reaction Buffer and 1.5 $\mu \mathrm{L}$ Bst DNA Polymerase, Large Fragment, with incubation at $65^{\circ} \mathrm{C}$ for 20 minutes followed by $80^{\circ} \mathrm{C}$ for 20 minutes. Libraries were purified with a MinElute column following the manufacturer's instructions and eluted in $25 \mu \mathrm{L}$ EB.

Method 4: "Ultra", in reference to NEBNext Ultra kit E7370. End-repair was conducted in a $0.5 \mathrm{~mL}$ Eppendorf LoBind tube using $13.9 \mu \mathrm{L}$ sample DNA, $1.6 \mu \mathrm{L}$ 10× NEBNext End Repair Reaction Buffer, and 0.75 $\mu \mathrm{L}$ NEBNext End Prep Enzyme Mix. The reaction was incubated for 30 minutes at $20^{\circ} \mathrm{C}$ followed by 30 minutes at $65^{\circ} \mathrm{C}$ and cooled to $4^{\circ} \mathrm{C}$. Subsequently, $1 \mu \mathrm{L}$ ATDC3 adapter $(10 \mu \mathrm{M})$ was added and mixed, followed by $3.75 \mu \mathrm{L}$ NEBNext Blunt/TA Ligase Master Mix and 0.25 $\mu \mathrm{L}$ NEBNext Ligation Enhancer. The reaction was incubated for 30 minutes at $20^{\circ} \mathrm{C}$. The fill-in reaction was done by adding a mastermix of $0.3 \mu \mathrm{L}$ dNTP (25 mM), $3 \mu \mathrm{L}$ Isothermal Amplification Buffer (10×) (NEB), $6.2 \mu \mathrm{L}$ molecular biology grade water, and 0.5 $\mu \mathrm{L}$ Bst 2.0 Warmstart Polymerase (NEB). The reaction was incubated at $65^{\circ} \mathrm{C}$ for 20 minutes in a prewarmed thermocycler, followed by 20 minutes at $80^{\circ} \mathrm{C}$. The reaction was purified with a MinElute column as described for method 1. 


\section{Quantitative PCR}

Quantitative PCR (qPCR) was performed on purified libraries with adaptertargeted primers (Supplementary, Table S1) using $1 \mu \mathrm{L}$ template of a 10× dilution of the libraries and a PCR mastermix consisting of 1x AmpliTaq Gold buffer (Applied Biosystems, USA), $2.5 \mathrm{mM} \mathrm{MgCl} 2,0.8 \mu \mathrm{g} / \mu \mathrm{L}$ Bovine Serum Albumin (BSA), $1 \mu \mathrm{L}$ SYBR/ROX/DMSO stock solution, $0.25 \mathrm{mM}$ dNTP, $0.2 \mu \mathrm{M}$ forward and reverse primer (IS7 and IS8 from Meyer \& Kircher, 2010), and 0.1 $\mathrm{U} / \mu \mathrm{L}$ AmpliTaq Gold enzyme to a final reaction volume of $25 \mu \mathrm{L}$. qPCR was performed on an Agilent Technologies Mx3005 instrument with the following cycling conditions: $95^{\circ} \mathrm{C}$ for 10 minutes, followed by 40 cycles of $95^{\circ} \mathrm{C}$ for 30 seconds, $60^{\circ} \mathrm{C}$ for 30 seconds, and $72^{\circ} \mathrm{C}$ for 1 minute. This was followed by $95{ }^{\circ} \mathrm{C}$ for one minute, $55^{\circ} \mathrm{C}$ for 30 seconds and $95^{\circ} \mathrm{C}$ for 30 seconds, to produce a dissociation curve (Supplementary, Figure S2).

\section{Indexing and PCR amplification of libraries for sequencing}

Libraries were indexed and amplified for sequencing using conventional fulllength P7 (indexed) and P5 Illumina primers (Supplementary, Table S1). PCR was performed in $50 \mu \mathrm{L}$ reactions using $10 \mu \mathrm{L}$ template, $1 \times$ AmpliTaq Gold buffer, $2.5 \mathrm{mM} \mathrm{MgCl} 2,0.8 \mu \mathrm{g} / \mu \mathrm{L}$ Bovine Serum Albumin (BSA), $0.25 \mathrm{mM} \mathrm{dNTP}$, $0.2 \mu \mathrm{M}$ forward and reverse indexed primer (specific for each sample), and 0.2 $\mathrm{U} / \mu \mathrm{L}$ AmpliTaq Gold polymerase. Libraries were amplified in an Applied Biosystems 2720 Thermal Cycler using the following conditions: $95^{\circ} \mathrm{C}$ for 10 minutes, followed by a number of cycles of $95^{\circ} \mathrm{C}$ for 30 seconds, $60^{\circ} \mathrm{C}$ for 30 
seconds, and $72{ }^{\circ} \mathrm{C}$ for 1 minute, followed by 7 minutes at $72^{\circ} \mathrm{C}$. Each library was given a specific number of PCR cycles based on $\mathrm{C}_{t}$ values from $\mathrm{qPCR}$ (Supplementary, Table S3). Quantification and size estimation was performed with an Agilent 2100 Bioanalyzer high sensitivity kit.

\section{Sequencing}

Sequencing was performed at the Danish National High-throughput Sequencing Center, Copenhagen, Denmark, on an Illumina HiSeq 2500 for 100 cycles in single read mode.

\section{Data Processing}

The initial bioinformatics processing of the sequencing reads was based on the Paleomix pipeline (Schubert et al. 2014). 3' adapter removal was performed with the program AdapterRemoval 2 (Schubert et al. 2016), followed by discarding reads shorter than 25 bases. The remaining reads were mapped to a reference genome - the de novo wolf genome (Gopalakrishnan et al. in review, available on request), using the mem algorithm implemented in BWA (Li \& Durbin, 2010). Reads that did not map uniquely were discarded. We used Picard (http://broadinstitute.github.io/picard) to discard PCR duplicates. Sequencing statistics and presented results were based on the obtained amount of filtered raw reads from sequencing. However, to verify that the disparate number of total sequencing reads did not bias the comparisons between the library preparation methods, we also performed the same analyses by subsampling the reads such that all the different samples and methods had exactly the same number of reads 
(Supplementary, Table S6). For each sample, we used 100,000 randomly selected reads to estimate the DNA damage patterns using mapDamage (Jónsson et al. 2013). We also used the package Preseq (Daley \& Smith, 2013) on the entire dataset to estimate the complexity of each library preparation method. Statistical tests were carried out in R (version 3.2.1, R Core Team, 2015) (Supplementary, Table S5).

\section{Results}

\section{$q P C R$ amplification and indexing $P C R$}

qPCR was used to assess the minimum number of PCR cycles required to amplify each library to the appropriate level for subsequent sequencing. The qPCR results also provided relative quantification of the number of library molecules present in each library.

Figure 2A shows that the BEMC method required most PCR cycles in order to reach the required threshold. Fewer cycles were required for the Ultra, BEST and ATST methods, with no significant difference between either of the singletube libraries (paired t-test $p>0.05$, Bonferroni corrected). $P$-values for statistical tests can be seen in supplementary Table S5.

Based on the $q \mathrm{PCR} \mathrm{C}_{t}$ values, each library was amplified with a given number of cycles with full-length Illumina primers. Because the difference in cycle number between qPCR and index PCR for all samples was almost the same ( $\sim 3$ cycles) (Supplementary, Table S3), we argue a comparison of DNA concentrations of amplified libraries is justified (Figure 2B). The concentration of amplified library 
differed between methods, with the ATST method giving significantly more PCR product than the BEMC method and the Ultra method (paired t-test $p<0.05$, Bonferroni corrected) (Supplementary, Table S3 and S5). Dissociation analysis of qPCR products showed a distinct difference between sample libraries and blanks, and size estimation of indexed PCR products further showed that adapter-dimer only was detectable in blanks (Supplementary, Figures S2-7). We argue this justifies the use of $\mathrm{qPCR}$ and PCR product quantification as measures of library preparation success.

\section{Effect on clonality and mapping success against reference genome}

Clonality (the percentage of reads that map to identical coordinates in the genome) was significantly lower for all single-tube libraries compared to the BEMC (Figure 3A, paired t-test $p<0.05$, Bonferroni corrected), and with no significant difference observed between single-tube library methods.

A major concern for most ancient and degraded DNA studies is the percentage of sequence reads that uniquely map to the reference genome of interest, because the percentage of endogenous DNA in ancient and degraded samples is often low and more damaged than the non-endogenous DNA (e.g. Meyer et al. 2016). The endogenous DNA content returned by the methods ranged between $40 \%$ and $53 \%$ (Figure 3B). All single-tube libraries showed significantly more reads mapping uniquely to the reference genome compared to the BEMC libraries (paired t-test $p<0.05$, Bonferroni corrected).

This article is protected by copyright. All rights reserved. 


\section{GC content}

Overall GC content was measured as an average for reads prior to mapping (Figure 3C). This showed that the GC content was significantly lower for the ATST compared to BEST and BEMC, respectively (paired t-test $p<0.05$, Bonferroni corrected).

\section{Read length}

To investigate the differences on retrieval and incorporation of short fragments between methods we estimated the average read lengths of the four library methods (Figure 3D). Surprisingly, we found a small but significant difference between BEST and BEMC, with BEST reads being shortest. Also, reads produced with the ATST method were significantly shorter than reads produced with the Ultra method (paired t-test $p<0.05$, Bonferroni corrected).

\section{Library complexity}

We used the Preseq package (Daley \& Smith, 2013) to estimate library complexity, which ultimately forecasts how deep a sample may be sequenced before the majority of reads generated are duplicates. The Preseq analysis clearly showed that the BEST method had the highest complexity on average, followed by ATST and Ultra, while the BEMC showed very low complexity (Figure 4).

This article is protected by copyright. All rights reserved. 


\section{Sequence-end bias}

To investigate sequence-bias for each library method, we also calculated single-nucleotide frequencies as an average for each method for the ten 5 ' bases upstream of the read, first ten bases within the read, last ten bases within the read (5'-3') and ten bases 3' downstream of the read (Figure 5). A clear pattern of increased A and G prior to the 5' end of the read is visible, consistent with previous aDNA findings (Briggs et al. 2007).

Also, consistent with the expectation for degraded DNA, we observed a high level of $\mathrm{C} \rightarrow \mathrm{T}$ transitions at the sequence $5^{\prime}$ ends, and a similar increase in $\mathrm{G} \rightarrow \mathrm{A}$ transitions at the 3' ends. This pattern has been shown to be a result of cytosine deamination of single-stranded overhangs in combination with the $3^{\prime}-5$ ' exonuclease activity and 5'-3' polymerase activity of the T4 DNA Polymerase during end repair (Briggs et al. 2007; Brotherton et al. 2007). Furthermore, for the first base, all single-tube libraries clearly show a sharp drop in thymine content towards the 5' end, breaking the pattern of increasing $\mathrm{C} \rightarrow \mathrm{T}$ transitions towards this end. At the same time, all other bases (A, G and C) exhibit an increase in frequency at this position in single-tube libraries, consistent with previous findings for A/T-ligation libraries (Seguin-Orlando et al. 2013). It should be noted that despite having fewer $\mathrm{C} \rightarrow \mathrm{T}$ transitions at the most extreme 5 ' base, all single-tube libraries seem to incorporate more $\mathrm{C} \rightarrow \mathrm{T}$ transitions at the penultimate 5' base.

This article is protected by copyright. All rights reserved. 


\section{Cost evaluation}

Finally, to evaluate the overall usefulness and practical implications of the different methods, we estimated the approximate cost and time required for producing 24 libraries using each method (Table 1), and found the ATST and BEST methods to have the lowest cost per library. We note that costs may vary if other enzyme suppliers or alternate purification strategies are used.

\section{Discussion and conclusion}

Results from qPCR and index PCR showed that the single-tube methods amplified earlier than the BEMC method, indicating a higher number of library molecules produced in the library preparation. Consequently, more library molecules likely resulted in lower clonality (Figure 3A), higher complexity (Figure 4) and higher rate of mapping success (Figure 3B).

This increase in production of library molecules for the single-tube methods might be explained by elimination of loss of target DNA during purifications, but also the altered enzymatic content. In previous protocols, a high concentration of

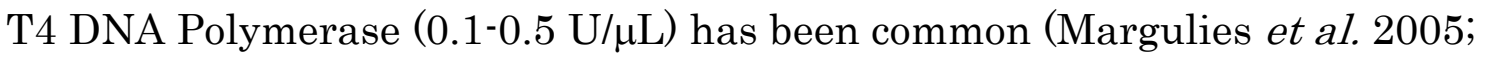
Kucera \& Nichols, 2008; Meyer et al. 2008; Meyer \& Kircher, 2010; Briggs \& Heyn, 2012). However, many enzyme suppliers (e.g. New England Biolabs, www.NEB.com) note that excessively high enzyme activity can lead to recessed 3' ends. Moreover, high temperatures are known to cause the same problem (Kucera \& Nichols, 2008). We speculate that this could be the case with the reagents used in the BEMC library preparation, given that this is designed for 
relatively high amounts of input DNA (1-5 $\mu \mathrm{g}, \mathrm{NEB})$ and therefore likely contains high enzyme concentrations. However, we emphasize that further testing is needed to investigate such a scenario.

Because the cost of sequencing increases with lower endogenous content, we argue that endogenous content of samples - here defined as the percentage of unique reads mapping to a reference genome - is one of the most important parameters when analyzing library methods for degraded DNA. Our results show that the single-tube methods and their efficiency have a large effect on the percentage of reads mapping to the reference genome. Consequently, the choice of library preparation method may greatly affect the quality of data, but also the costs and efficiency of sequencing efforts. Furthermore, suboptimal library preparation efficiency during screening of samples may lead to "false negative" results and subsequent oversampling from the original tissues sampled.

GC content is recognized as an important parameter for sequencing libraries and has been shown to be highly affected by PCR (e.g. Aird et al. 2011; Dabney \& Meyer, 2012; Quail et al. 2012). Although we found no significant difference between Ultra libraries and BEST/BEMC, there was a significant difference between ATST and BEST/BEMC. Our results do not clearly point to a cause inherent to A/T-ligation or to the single-tube design, and because this parameter is heavily affected by PCR, we believe that further studies are needed to elucidate any differences in GC content arising specifically in the library build. The read length is also often used to evaluate library preparation on degraded DNA (e.g. Bennett et al. 2014; Gansauge \& Meyer, 2017; Wales et al. 2015). Although our results do not solely point to single-tube methods retrieving shorter 
fragments as such, it is intriguing that heat inactivation at $65^{\circ}$ does not increase the average read lengths as could be expected due to denaturation of short fragments (Owczarzy et al. 1997). In fact, with the higher number of indexing PCR cycles given to the BEMC libraries (Supplementary, Table S3) and expected related decrease in average read length (Dabney \& Meyer, 2012) the true difference between methods before PCR amplification might even be greater. Arguably, many of the above factors will affect the library complexity. This has large implications for sequencing degraded samples in which little DNA material is present and massive sequencing is needed due to the often low endogenous content and short read lengths (see e.g. Meyer et al. 2016). Our estimate on library complexity using the Preseq package (Daley \& Smith, 2013) showed that all single tube preparations had higher complexity than the BEMC method. For very degraded samples, or samples with limited amount of sampled material, such a parameter would have a great impact on the ability to obtain high quality genetic data, as previously shown with the single-stranded library method (Meyer et al. 2012).

Sequence nucleotide composition analysis (Figure 5) showed that damage patterns caused by cytosine deamination were significantly higher for the BEMC library at the terminal 5' position. It could be argued that this shows a stronger tendency of BEMC to incorporate endogenous DNA fragments (Skoglund et al. 2014; Meyer et al. 2016). However, this scenario seems unlikely considering the lower percentage of reads mapping to the reference genome for the BEMC library (Figure 3B). One explanation for this discrepancy could be that the number of fragments that actually contain a uracil base at the 5 ' terminal 
position constitute a small percentage of the total number of fragments.

Therefore, the results do not point solely towards lower incorporation of damaged molecules overall, but more specifically, lower incorporation of molecules bearing uracil or thymine at the terminal 5' position. Moreover, the negative effect of this phenomenon could be overruled by an overall more efficient library preparation, which results in higher complexity and read mapping. Therefore, regardless of using blunt-end or A/T-ligation, single-tube preparation seems to be beneficial for degraded DNA.

We propose that the observed pattern in all single-tube methods in which $\mathrm{C} \rightarrow \mathrm{T}$ transitions increase in frequency towards the 5 ' end, but with a sudden drop at the 5 ' terminal base, most likely point to a bias caused by sequence-dependent enzymatic substrate preference as previously shown for A/T-ligation (SeguinOrlando et al. 2013). However, this bias might be intrinsic to more than one enzyme used in the presented methods. The effect is strongest in the libraries with A/T-ligation, but is also seen in the BEST libraries (Figure 5). Because much lower concentrations of enzymes (especially the T4 DNA polymerase) were used in the BEST method compared to the BEMC, we propose an explanation in which the concentration of T4 DNA polymerase can act as a double-edged sword where high concentrations cause an inefficient library preparation due to recessed 3' ends (as previously mentioned), while low concentrations can enhance the intrinsic bias of the enzyme. However, we emphasize that this scenario should be further tested in more rigorous experiments. Although the four presented methods share many enzymes, it should also be noted that the fill-in 
reaction differs in its use of "Bst DNA Polymerase, Large Fragment" for BEMC and "Bst DNA Polymerase 2.0" for Ultra, BEST and ATST.

It is difficult to establish the precise biochemical causes of the downstream sequencing results and it must be emphasized that the presented data are a result of a number of steps in combination and that further studies are needed to elucidate the details. However, the effect on sequencing costs is clear when using single-tube design, given that less sequencing is needed to obtain a given coverage of the target sample. Importantly, the single-tube design allows for a low-cost and easy parallelization of library preparation (automated or manual) as previously shown for modern DNA (e.g. Meyer \& Kircher, 2010; Rohland \& Reich, 2012). The presented single-tube methods therefore enable efficient highthroughput library preparation on very short and degraded DNA fragments. Due to the simplicity, low cost, high complexity and the slightly lower bias signal compared to the ATST and Ultra, we regard the BEST method to be the most suitable single-tube protocol for degraded DNA.

From a technical perspective, the present study has yielded insight into the possible mechanisms behind library bias, although we emphasize that further studies are needed to elucidate the specific causes of the observed patterns. From a practical perspective and especially in regards to the field of aDNA and related fields, the presented results and methodologies demonstrate the possibility and benefits of building high quality sequencing libraries from degraded DNA, in a time- and cost-effective manner. 


\section{Acknowledgements}

We would like to acknowledge the Advanced Technology Foundation

"FoodGenomics” Grant (080-2012-3), DFF “Wineometrics” grant (4005-00107), Marie Skłodowska-Curie Individual Fellowships (IF-EF) "WhereWolf" (655732), ERC Consolidator "Extinction Genomics”Grant (681396) and The Danish National Research Foundation (DNRF94) for funding and the Danish National High-Throughput Sequencing Centre for assistance in data generation. We are grateful to members of the FoodGenomics project for comments during the development of this work. We would also like to thank the Natural history Museum of Denmark, in particular Kristian Murphy Gregersen and Mogens Andersen for access to sample collections.

\section{Data accessibility}

The data is accessible at the Electronic Research Data Archive at the University of Copenhagen (UCPH ERDA).

http://www.erda.dk/public/archives/YXJjaGl2ZS1McldVYkU=/publishedarchive.html

\section{Conflict of interest}

The authors declare no conflict of interest.

\section{Author contributions}

CC, LV, MTPG and TSP conceived the ideas and designed methodology. MHSS collected data. CC, MHSS and SSTM performed laboratory experiments. CC, SG, 
NW and JAS analyzed the data. CC led the writing of the manuscript. All

authors contributed critically to the draft and gave final approval for publication.

\section{References}

Aird D, Ross MG, Chen W-S et al. (2011) Analyzing and minimizing PCR amplification bias in Illumina sequencing libraries. Genome Biology, 12:R18

Allentoft ME, Sikora M, Sjögren K-G et al. (2015) Population genomics of Bronze Age Eurasia. Nature, 522, 167-172.

Bennett EA, Massilani D, Lizzo G et al. (2014) Library construction for ancient genomics: Single strand or double strand? BioTechniques, 56, 289-300.

Bentley DR, Balasubramanian S, Swerdlow HP et al. (2008) Accurate whole human genome sequencing using reversible terminator chemistry. Nature, 456, 53-59.

Briggs AW, Heyn P (2012) Preparation of Next-Generation Sequencing Libraries from Damaged DNA. Ancient DNA: Methods and Protocols, Methods in Molecular Biology, $840,143-154$

Briggs AW, Stenzel U, Johnson PLF et al. (2007) Patterns of damage in genomic DNA sequences from a Neandertal. Proceedings of the National Academy of Sciences of the United States of America, 104, 14616-14621.

Briggs AW, Stenzel U, Meyer M et al. (2010) Removal of deaminated cytosines and detection of in vivo methylation in ancient DNA. Nucleic Acid Research, 38, no. 6.

Brotherton P, Endicott P, Sanchez JJ et al. (2007) Novel high-resolution characterization of ancient DNA reveals $\mathrm{C}>\mathrm{U}$-type base modification events as the sole cause of post mortem miscoding lesions. Nucleic Acids Research, 35, 5717-5728.

d'Abbadie M, Hofreiter M, Vaisman A et al. (2007) Molecular breeding of polymerases for amplification of ancient DNA. Nature Biotechnology, 25, 939-943.

Dabney J, Knapp M, Glocke I et al. (2013a) Complete mitochondrial genome sequence of a Middle Pleistocene cave bear reconstructed from ultrashort DNA fragments. Proceedings of the National Academy of Sciences of the United States of America, 110, 15758-15763.

This article is protected by copyright. All rights reserved. 
Dabney J, Meyer M (2012) Length and GC-biases during sequencing library amplification: A comparison of various polymerase-buffer systems with ancient and modern DNA sequencing libraries. BioTechniques, 52, 87-94.

Dabney J, Meyer M, Pääbo S (2013b) Ancient DNA Damage. Cold Spring Harb Perspect Biol, 5, $1-7$.

Daley T, Smith AD (2013) Predicting the molecular complexity of sequencing libraries. Nature Methods, 10, 325-327.

Damgaard PB, Margaryan A, Schroeder H et al. (2015) Improving access to endogenous DNA in ancient bones and teeth. Scientific Reports, 5, 11184.

Van Dijk EL, Jaszczyszyn Y, Thermes C (2014) Library preparation methods for next-generation sequencing: Tone down the bias. Experimental Cell Research, 322, 12-20.

Fortes GG, Paijmans JLA (2015) Analysis of whole mitogenomes from ancient samples. In: Whole Genome Amplification: Methods and Protocols, pp. 179-195.

Gamba C, Hanghøj K, Gaunitz C et al. (2015) Comparing the performance of three ancient DNA extraction methods for high-throughput sequencing. Molecular Ecology Resources, 16, 459469.

Gansauge M-T et al. (2017) Single-stranded DNA library preparation from highly degraded DNA using T4 DNA ligase. Nucleic Acid Research. doi: 10.1093/nar/gkx033

Gansauge M-T, Meyer M (2013) Single-stranded DNA library preparation for the sequencing of ancient or damaged DNA. Nature Protocols, 8, 737-748.

Gansauge M-T, Meyer M (2014) Selective enrichment of damaged DNA molecules for ancient genome sequencing, Genome research, 24, 1543-1549.

Ginolhac A, Rasmussen M, Gilbert MTP, Willerslev E, Orlando L (2011) mapDamage: testing for damage patterns in ancient DNA sequences. Bioinformatics (Oxford, England), 27, 21532155.

Ginolhac A, Vilstrup J, Stenderup J et al. (2012) Improving the performance of true single molecule sequencing for ancient DNA. BMC Genomics, 13, 177.

Head SR, Kiyomi Komori H, LaMere SA et al. (2014) Library construction for next-generation sequencing: Overviews and challenges. BioTechniques, 56, 61-77.

This article is protected by copyright. All rights reserved. 
Heintzman PD, Soares AER, Chang D, Shapiro B (2015) Paleogenomics. Reviews in Cell Biology and Molecular Medicine, 1, 243-267.

Heyn P, Stenzel U, Briggs AW et al. (2010) Road blocks on paleogenomes-polymerase extension profiling reveals the frequency of blocking lesions in ancient DNA. Nucleic Acids Research, 38, $\mathrm{e} 161$.

Jónsson H, Ginolhac A, Schubert M, Johnson PLF, Orlando L (2013) MapDamage2.0: Fast approximate Bayesian estimates of ancient DNA damage parameters. Bioinformatics, 29, $1682-1684$.

Kemp BM, Winters M, Monroe C, Barta JL (2014) How Much DNA is Lost? Measuring DNA Loss of STR Length Fragments Targeted by the PowerPlex 16® System Using the Qiagen MinElute Purification Kit. Human Biology Open Access Pre-Prints, Paper 61.

Kucera RB, Nichols NM (2008) DNA-dependent DNA polymerases. Current Protocols in Molecular Biology, 1-19.

Li H, Durbin R (2010) Fast and accurate long-read alignment with Burrows-Wheeler transform. Bioinformatics, 26, 589-595.

Margulies M, Egholm M, Altman WE et al. (2005) Genome sequencing in microfabricated highdensity picolitre reactors. Nature, 437, 376-80.

Maricic T, Pääbo S (2009) Optimization of 454 sequencing library preparation from small amounts of DNA permits sequence determination of both DNA strands. BioTechniques, 46, $51-57$.

Meyer M, Arsuaga J-L, de Filippo C et al. (2016) Nuclear DNA sequences from the Middle Pleistocene Sima de los Huesos hominins. Nature, 531, 504-507.

Meyer M, Kircher M (2010) Illumina sequencing library preparation for highly multiplexed target capture and sequencing. Cold Spring Harbor Protocols, doi:10.1101/pdb.prot5448.

Meyer M, Kircher M, Gansauge M et al. (2013) A High Coverage Genome Sequence From an Archaic Denisovan Individual, Science, 338, 222-226

Meyer M, Stenzel U, Hofreiter M (2008) Parallel tagged sequencing on the 454 platform. Nature protocols, 3, 267-278.

Neiman M, Sundling S, Grönberg H et al. (2012) Library Preparation and Multiplex Capture for

This article is protected by copyright. All rights reserved. 
Massive Parallel Sequencing Applications Made Efficient and Easy. PLoS ONE, 7, 7-12.

Orlando L, Gilbert MTP, Willerslev E (2015) Reconstructing ancient genomes and epigenomes. Nature Reviews Genetics, 16, 395-408.

Owczarzy R, Vallone PM, Gallo FJ et al. (1997) Predicting sequence-dependent melting stability of short duplex DNA oligomers. Biopolymers, 44, 217-239.

Poinar HN, Schwarz C, Qi J et al. (2006) Metagenomics to paleogenomics: large-scale sequencing of mammoth DNA. Science, 311, 392-394.

MA, Otto TD, Gu Y, Harris SR et al. (2012) Optimal enzymes for amplifying sequencing libraries. Nature Methods, 9, 10-11.

R Core Team (2015). R: A language and environment for statistical computing Foundation for Statistical Computing, Vienna, Austria. URL http://www.R-project.org/.

Rohland N, Hofreiter M (2007) Ancient DNA extraction from bones and teeth. Nature Protocols, $2,1756-1762$.

Rohland N, Reich D (2012) Cost-effective, high-throughput DNA sequencing libraries for multiplexed target capture. Genome Research, 22, 939-946.

Schubert M, Ermini L, Der Sarkissian C et al. (2014) Characterization of ancient and modern genomes by SNP detection and phylogenomic and metagenomic analysis using PALEOMIX. Nature Protocols, 9, 1056-1082.

Schubert M, Lindgreen S, Orlando L (2016) AdapterRemoval v2: rapid adapter trimming, identification, and read merging. BMC Research Notes, $\mathbf{9}, 88$.

Seguin-Orlando A, Schubert M, Clary J et al. (2013) Ligation bias in illumina next-generation DNA libraries: implications for sequencing ancient genomes. PloS ONE, 8, e78575.

Shapiro B (2008) Engineered polymerases amplify the potential of ancient DNA. Trends in Biotechnology, 26, 285-287.

Shapiro B, Hofreiter M (2014) A paleogenomic perspective on evolution and gene function: new insights from ancient DNA. Science, 343, 1236573.

Skoglund P, Northoff BH, Shunkov MV et al. (2014) Separating endogenous ancient DNA from modern day contamination in a Siberian Neandertal, Proceedings of the National Academy of Sciences of the United States of America, 6, 2229-34.

This article is protected by copyright. All rights reserved. 
Wales N, Carøe C, Sandoval-Velasco M et al. (2015) New insights on single-stranded versus double-stranded DNA library preparation for ancient DNA. BioTechniques, 59, 368-371.

Zheng Z, Advani A, Melefors O et al. (2011) Titration-free 454 sequencing using Y adapters.

Nature Protocols, 6, 1367-1376.

Tables

\begin{tabular}{|c|c|c|c|c|c|c|c|}
\hline Method & $\begin{array}{c}\text { Ligation } \\
\text { type }\end{array}$ & $\begin{array}{c}\text { Reaction } \\
\text { design }\end{array}$ & $\begin{array}{c}\text { Adapters } \\
\text { used }\end{array}$ & Reagents reference & Cost (\$) & $\begin{array}{c}\text { Total time for 24 } \\
\text { samples (hours) }\end{array}$ & $\begin{array}{c}\text { Hands-on time } \\
\text { for 24 samples } \\
\text { (hours) }\end{array}$ \\
\hline ATST & A/T & Single-tube & ATDC3 & This study & $\begin{array}{c}3.78 \\
(0.76)\end{array}$ & 4 & 2 \\
\hline BEST & Blunt-end & Single-tube & BEDC3 & This study & $\begin{array}{c}3.77 \\
(0.77)\end{array}$ & 4 & 2 \\
\hline BEMC & Blunt-end & 3 reaction & $\begin{array}{c}\text { Keyer \& } \\
\text { Kircher, }\end{array}$ & NEB \#E6070 & 14.32 & 6 & 4 \\
\hline Ultra & A/T & Single-tube & ATDC3 & NEB Ultra \#E7370 & $\begin{array}{c}8.98 \\
(5.97)\end{array}$ & & 4 \\
\hline
\end{tabular}

Table 1. An overview of the methods used in the present study and their characteristics.

Estimated prices are listed in US Dollars (\$) and calculated based on how they are presented in this paper, including consumables, enzymes, and purification spin columns. Prices in parentheses represent substitution of MinElute with SPRI beads as presented in Rohland \& Reich (2012). Adapters were estimated to cost $\sim \$ 0.10$ per reaction. Because we only used $1 / 4$ of the reaction volume for ultra and BEMC libraries, this price is scaled down relative to the price for a whole reaction given by NEB. The price for the NEB \#E6070 kit, used for the BEMC method, is calculated using two MinElute columns although we used 3 to ensure proper comparison and similar conditions for all methods in downstream PCR. Oligos used for the adapters listed in column 4, can be found in supplementary, Table S1.

1)

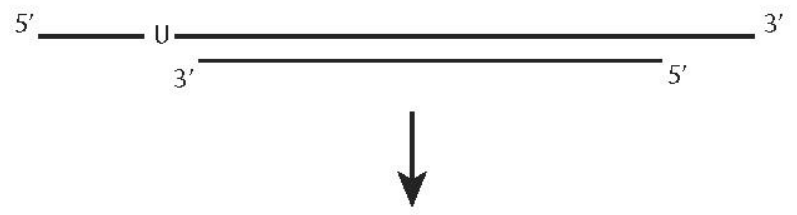

2)

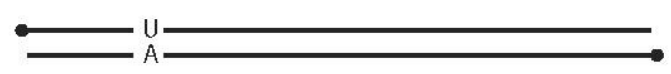

3)
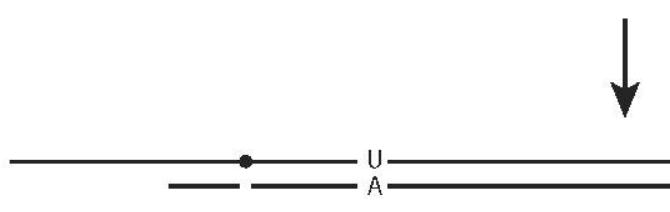

4)

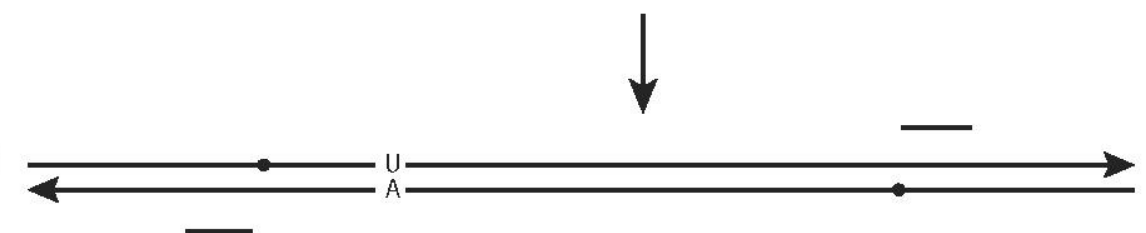

This article is protected by copyright. All rights reserved. 

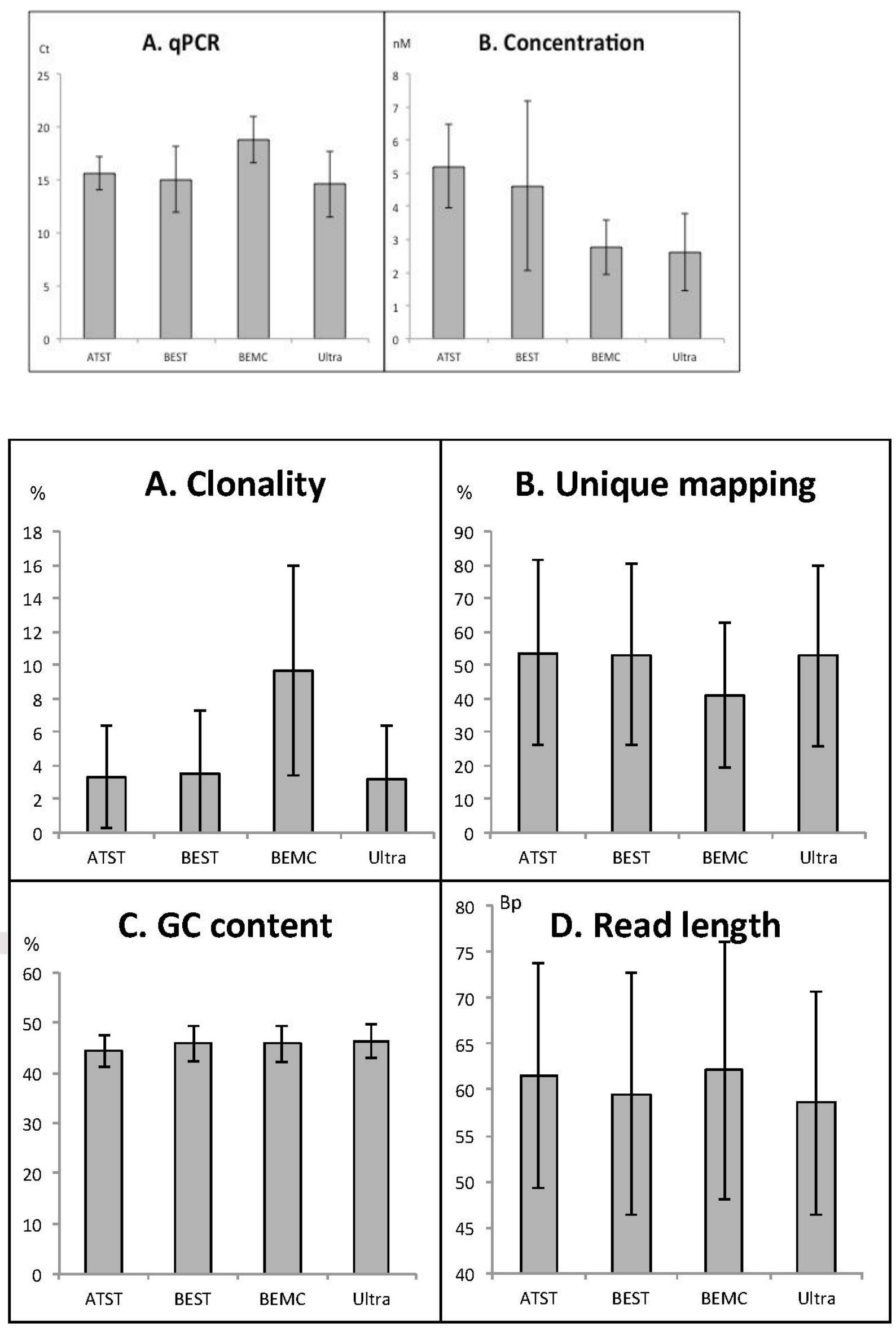

This article is protected by copyright. All rights reserved. 


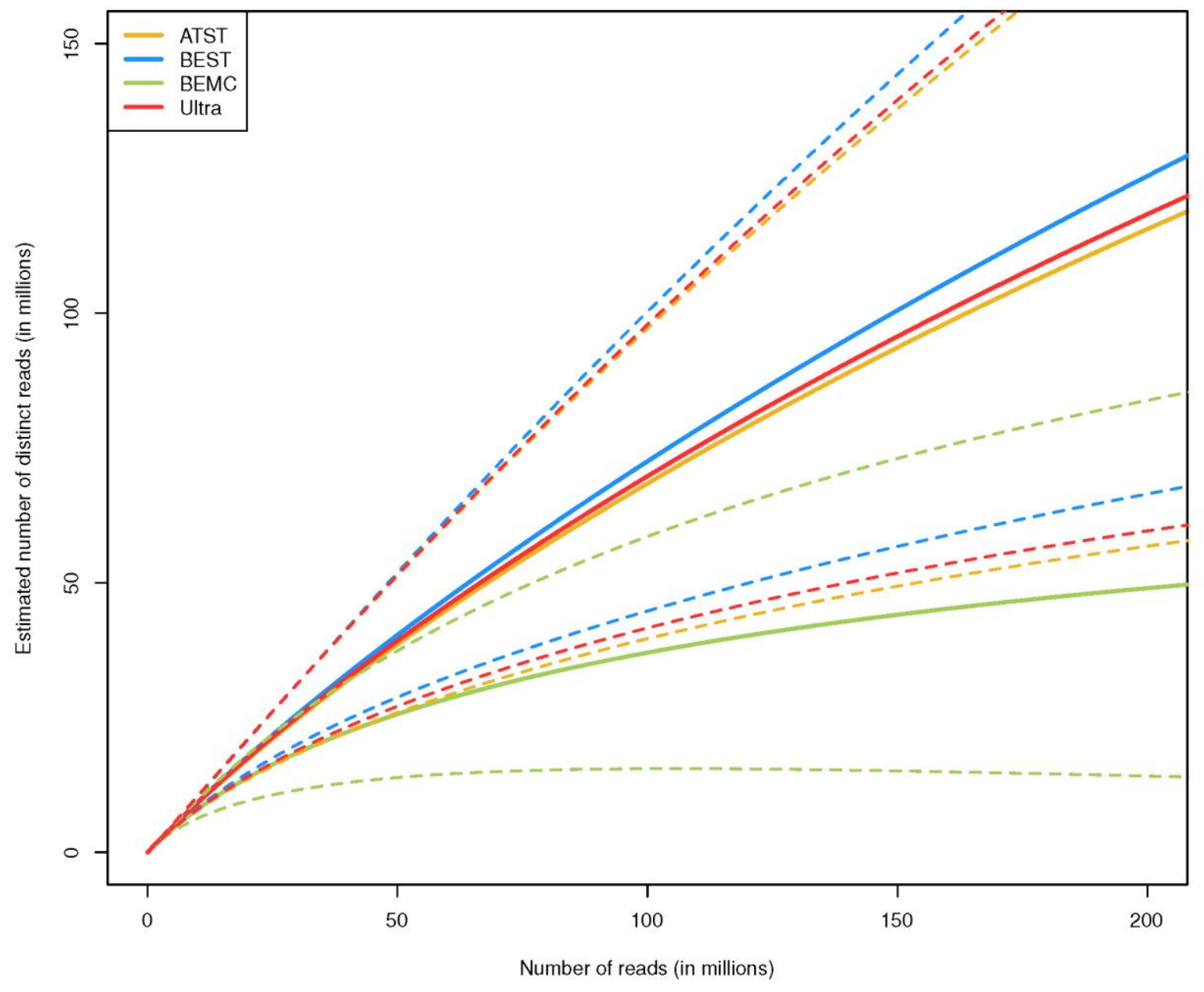

This article is protected by copyright. All rights reserved. 

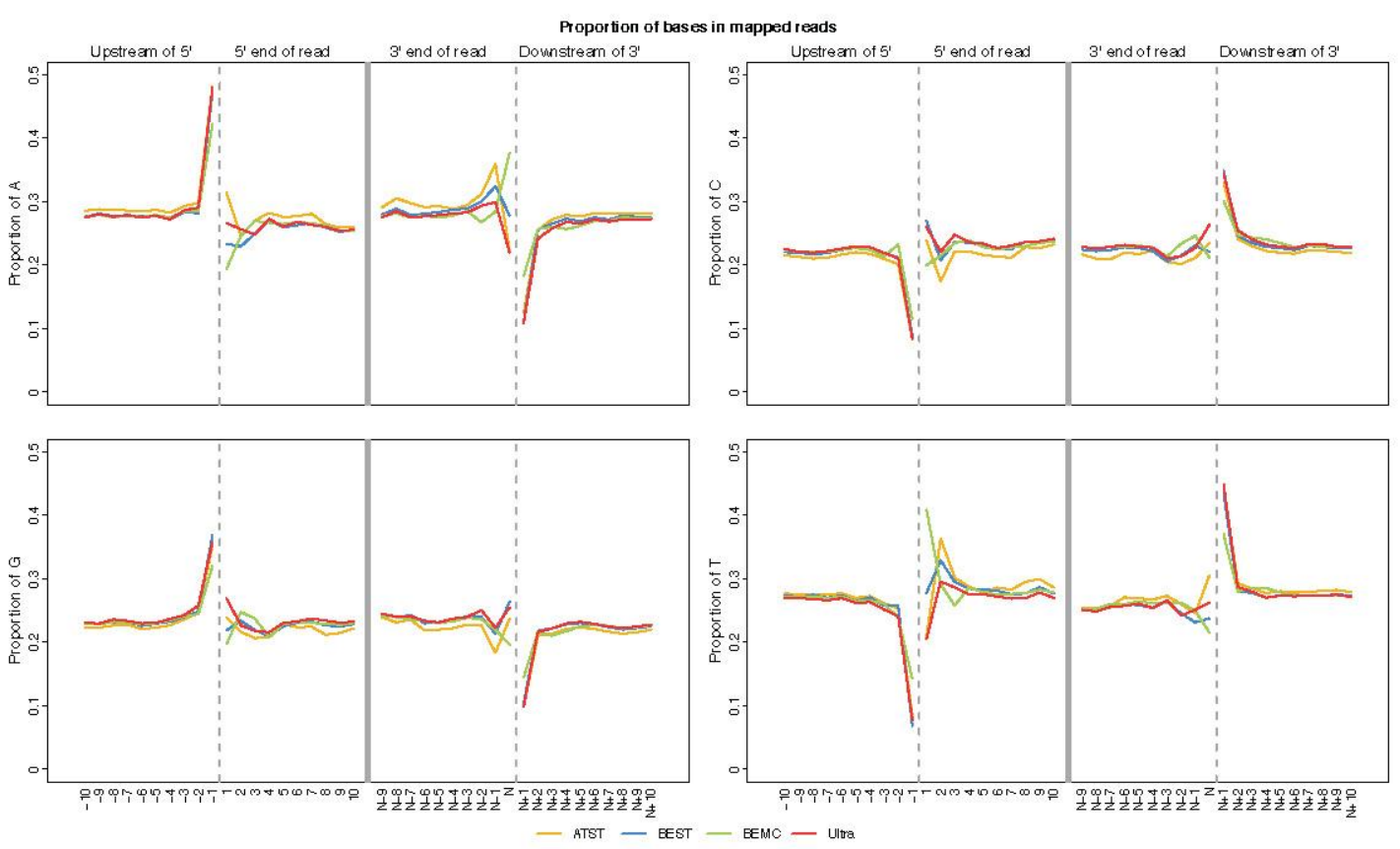

This article is protected by copyright. All rights reserved. 